\title{
Characteristics and Outcomes of Patients Undergoing Endoscopy During the COVID-19 Pandemic: A Multicenter Study from New York City
}

\author{
John W. Blackett ${ }^{1} \cdot$ Nikhil A. Kumta $^{2} \cdot$ Rebekah E. Dixon $^{2} \cdot$ Yakira David $^{2} \cdot$ Satish Nagula $^{2} \cdot$ Christopher J. DiMaio $^{2}$. \\ David Greenwald ${ }^{2} \cdot$ Reem Z. Sharaiha ${ }^{3} \cdot$ Kartik Sampath $^{3}$ - David Carr-Locke ${ }^{3}$. Arcelia Guerson-Gil ${ }^{4} \cdot$ Sammy Ho $^{4}$. \\ Benjamin Lebwohl ${ }^{1} \cdot$ Reuben Garcia-Carrasquillo $^{1}$ - Anjana Rajan ${ }^{3}$. Vasantham Annadurai ${ }^{1}$. Tamas A. Gonda ${ }^{1}$. \\ Daniel E. Freedberg ${ }^{1} \cdot$ Srihari Mahadev ${ }^{3}$ (1)
}

Received: 22 June 2020 / Accepted: 28 August 2020 / Published online: 15 September 2020

○) Springer Science+Business Media, LLC, part of Springer Nature 2020

\begin{abstract}
Background The coronavirus disease 2019 (COVID-19) pandemic has significantly impacted the practice of endoscopy, but characteristics of COVID patients undergoing endoscopy have not been adequately described.

Aims To compare findings, clinical outcomes, and patient characteristics of endoscopies performed during the pandemic in patients with and without COVID-19.

Methods This was a retrospective multicenter study of adult endoscopies at six academic hospitals in New York between March 16 and April 30, 2020. Patient and procedure characteristics including age, sex, indication, findings, interventions, and outcomes were compared in patients testing positive, negative, or untested for COVID-19.

Results Six hundred and five endoscopies were performed on 545 patients during the study period. There were 84 (13.9\%), 255 (42.2\%), and 266 (44.0\%) procedures on COVID-positive, negative, and untested patients, respectively. COVID patients were more likely to undergo endoscopy for gastrointestinal bleeding or gastrostomy tube placement, and COVID patients with gastrointestinal bleeding more often required hemostatic interventions on multivariable logistic regression. COVID patients had increased length of stay, intensive care unit admission, and intubation rate. Twenty-seven of 521 patients (5.2\%) with no or negative COVID testing prior to endoscopy later tested positive, a median of 13.5 days post-procedure.

Conclusions Endoscopies in COVID patients were more likely to require interventions, due either to more severe illness or a higher threshold to perform endoscopy. A significant number of patients endoscoped without testing were subsequently found to be COVID-positive. Gastroenterologists in areas affected by the pandemic must adapt to changing patterns of endoscopy practice and ensure pre-endoscopy COVID testing.
\end{abstract}

Keywords COVID-19 · Coronavirus · Endoscopy · Gastrointestinal bleeding

\section{Introduction}

Electronic supplementary material The online version of this article (https://doi.org/10.1007/s10620-020-06593-9) contains supplementary material, which is available to authorized users.

Srihari Mahadev

srm9005@med.cornell.edu

1 Division of Digestive and Liver Diseases, Columbia University Medical Center, New York, NY, USA

2 Division of Gastroenterology, Icahn School of Medicine at Mount Sinai, New York, NY, USA
The novel coronavirus pandemic brought routine endoscopy in many areas of the world to a near halt, particularly in New York City, one of the major epicenters of the crisis in

3 Division of Gastroenterology and Hepatology, Weill Cornell Medical Center, 1305 York Ave, 4th Floor, New York, NY 10065, USA

4 Division of Gastroenterology and Liver Diseases, Montefiore Medical Center, New York, NY, USA 
early 2020 [1]. It has disrupted scheduled cancer screenings, care of inflammatory bowel disease patients, and evaluation of iron deficiency anemia, among other common gastrointestinal problems [2,3]. Fellow education has also been adversely affected as a result of the sharp drop in endoscopy volume.

Nonetheless, patients have still been presenting with gastrointestinal hemorrhage, acute cholangitis, food impactions, and other urgent indications for endoscopy that cannot wait until the pandemic has subsided. Endoscopy in patients with coronavirus disease 2019 (COVID-19) requires careful patient selection, balancing the risks of staff exposure and use of protective equipment against the benefits of potentially life-saving endoscopic interventions [4-8]. There are few studies exploring the types of endoscopies performed during the COVID-19 pandemic. Given that cases are still rising at an alarming rate in the USA and the likelihood that the coronavirus pandemic will continue to affect the practice of endoscopy for the foreseeable future, it is important to understand how outcomes and findings differ in patients with COVID-19 compared to non-COVID patients [1].

The purpose of this multicenter study was to describe in detail the characteristics, findings, interventions, and outcomes of patients undergoing endoscopy during the peak of the COVID-19 pandemic in New York City, one of the global epicenters of the pandemic in early 2020 . We compared the endoscopies performed, need for intervention, and clinical outcomes in patients who had COVID-19 with those who were COVID-negative or were not tested. We hypothesized that COVID patients would have a higher diagnostic and therapeutic yield, because of a strategy to pursue supportive care or defer endoscopy in COVID patients with all but the most time-sensitive indications. We also measured the frequency with which patients who underwent endoscopy without COVID testing were subsequently found to be COVID-positive. Although the effects of the COVID-19 pandemic have begun to stabilize, many areas of the USA are still facing increasing numbers of cases. It is important to continue exercising caution in patient selection for endoscopy. Our experiences during this critical time may prove useful to gastroenterologists as they confront increasing numbers of COVID-19 patients in need of urgent endoscopic evaluation or intervention.

\section{Methods}

The electronic medical records of all adult patients who underwent endoscopy between March 16 and April 30, 2020 at six academic hospitals in New York City (the Allen Hospital, Columbia University Irving Medical Center, Lower Manhattan Hospital, Montefiore Medical Center, Mount Sinai Hospital, and Weill Cornell Medical Center) were reviewed. March 16 was selected as the start because that was the date after which routine endoscopies were canceled at all of the participating institutions. Both inpatients and outpatients were included. The results of all SARS-CoV-2 testing both before and after endoscopy were recorded. Patient and procedure characteristics including age, sex, procedure type, indication, anesthesia level, fellow involvement, findings, and interventions were analyzed. Outcomes including intensive care unit admission (at any time during hospitalization, including before endoscopy), intubation, length of stay, and death were also measured. For the 68 patients still admitted at the time of review $(20.6 \%$ of the total number of admitted patients), their length of stay was defined as length of stay through April 30, 2020. Stratified analyses were performed comparing patients with positive SARS-CoV-2 testing to those with negative or no testing, and inpatients to outpatients. Continuous variables were compared using the Mann-Whitney $U$ test for two groups and Kruskal-Wallis analysis of variance for more than two groups. Categorical variables were compared using the Chi-square test, or Fisher's exact test when at least $25 \%$ of expected cell counts were less than 5 . For patients who had multiple procedures on separate days during the time period, patients were only counted once for demographic characteristics and outcomes such as age, sex, and death, but each procedure was considered separately for endoscopy-specific characteristics such as anesthesia type, procedure type, and indication. Procedures done in the same session (such as an upper endoscopy and colonoscopy) were counted as a single procedure. Because this was a retrospective analysis of already collected data, informed consent was not required. The institutional review boards of Columbia University, Cornell University, Mount Sinai Hospital, and Montefiore Medical Center approved this study.

\section{Results}

\section{Population}

Between March 16, 2020 and April 30, 2020, 605 endoscopic procedures were performed on 545 patients at the six hospitals included in the study (Table 1). There were 383 inpatient procedures on 330 patients, and 222 outpatient procedures on 215 patients. Patient and endoscopy characteristics stratified by admission status are shown in Supplemental Table 1 and Supplemental Table 2, respectively. There were 84 endoscopies (13.9\%) performed on patients with a positive SARS-CoV-2 test either before or on the day of the procedure, compared to 255 (42.2\%) done with a confirmed negative test. There were 266 endoscopies $(44.0 \%)$ with no testing done at the time of the procedure. There were $260(43.0 \%)$ procedures in the last 2 weeks of 
Table 1 Characteristics of patients undergoing endoscopy during the COVID-19 pandemic

\begin{tabular}{|c|c|}
\hline Total number of patients & 545 \\
\hline Total number of procedures & 605 \\
\hline Median years of age (interquartile range) & $63(52-73)$ \\
\hline \multicolumn{2}{|l|}{ Sex } \\
\hline Female & $217(39.8 \%)$ \\
\hline Male & $328(60.2 \%)$ \\
\hline \multicolumn{2}{|l|}{ Admission status } \\
\hline Inpatient & $\begin{array}{c}383(63.3 \%) \\
\text { procedures } \\
\text { on } 330 \\
\text { patients }\end{array}$ \\
\hline Outpatient & $\begin{array}{l}222(36.7 \%) \\
\text { procedures } \\
\text { on } 215 \\
\text { patients }\end{array}$ \\
\hline \multicolumn{2}{|l|}{ COVID status at time of procedure } \\
\hline Negative & $255(42.2 \%)$ \\
\hline Positive & $84(13.9 \%)$ \\
\hline No testing & $266(44.0 \%)$ \\
\hline \multicolumn{2}{|l|}{ Month of procedure } \\
\hline March 16-March 31 & $260(43.0 \%)$ \\
\hline April 1-April 30 & $345(57.0 \%)$ \\
\hline \multicolumn{2}{|l|}{ Site } \\
\hline The Allen Hospital & $20(3.3 \%)$ \\
\hline Columbia University Irving Medical Center & $150(24.8 \%)$ \\
\hline Lower Manhattan Hospital & $23(3.8 \%)$ \\
\hline Mount Sinai Hospital & $172(28.4 \%)$ \\
\hline Montefiore Medical Center & $101(16.7 \%)$ \\
\hline Weill Cornell Medical Center & $139(23.0 \%)$ \\
\hline \multicolumn{2}{|l|}{ Outcomes among 330 admitted patients } \\
\hline Death & $28(8.5 \%)$ \\
\hline Admission to Intensive Care Unit & $128(38.8 \%)$ \\
\hline Intubation (not solely for procedure) & $84(25.5 \%)$ \\
\hline Median length of stay (interquartile range) & $11(4-23)$ \\
\hline
\end{tabular}

March, compared to 345 (57.0\%) done in April. There were 27 patients $(5.0 \%)$ who had either not been tested or tested negative at the time of their endoscopy, but subsequently tested positive for COVID-19. Among the 330 inpatients undergoing endoscopy during this period, 128 (38.8\%) were admitted to the intensive care unit, 84 were intubated at any point during hospitalization for reasons other than the endoscopy (25.5\%), and 28 died (8.5\%).

\section{Stratified by COVID-19 Status}

Among the 605 endoscopies during this period, 84 were on patients known to have COVID-19 (13.9\% of total, $24.8 \%$ of the 339 patients with COVID testing at time of endoscopy, see Table 2). Patients tested for COVID had a higher median age than those who were not tested (66 compared to 61.5 years). COVID-positive patients were more likely to be male (73.4\% compared to $59.3 \%$ of COVID-negative and $56.7 \%$ of untested patients). COVID-positive patients were far more likely to be inpatients $(91.1 \%)$ compared to COVID-negative (66.8\%) or untested patients (44.6\%). There were seven patients who had tested positive for COVID prior to an outpatient endoscopy. Three of these were upper endoscopies (including one for percutaneous endoscopic gastrostomy tube placement), three were endoscopic retrograde cholangiopancreatography (ERCP), and one was a colonoscopy. The indications were for dysphagia for two of the upper endoscopies and suspected stenosis of the gastrointestinal tract for the third. The indication for the ERCPs were suspected ascending cholangitis, abnormal imaging, and abnormal liver function tests. The indication for the colonoscopy was suspected colon mass on imaging. The majority of procedures done without COVID testing were in March (78.6\%), while 92.9\% of the COVID-positive procedures and $82.4 \%$ of the COVID-negative procedures were in April. Figure 1 shows the cumulative numbers of procedures done separated by COVID status at the time of endoscopy. Initially, almost all procedures were done without COVID testing, but over time nearly all patients began to be tested prior to endoscopy. There were initially very few endoscopies done on COVID-positive patients, but the number began to increase starting in mid-April.

\section{Outcomes in Hospitalized Patients}

Among the 330 hospitalized patients, COVID-positive patients were far more likely to require the intensive care unit, at $65.3 \%$ compared to $31.8 \%$ of COVID-negative patients and $30.8 \%$ of untested COVID patients. Similarly, COVID-positive patients were more likely to be intubated (for reasons not solely for the endoscopy). There was a higher mortality rate among COVID-positive patients at $11.1 \%$ compared to $6.6 \%$ in COVID-negative patients and 9.4\% in untested patients, but this difference was not statistically significant. The median length of stay was nearly twice as long among patients who were COVID-positive at the time of their endoscopy at 19 days, compared to 8 days in COVID-negative patients and 10 days in untested patients.

\section{Endoscopy Characteristics}

There were significant differences in the types of procedures performed in COVID-positive patients compared to COVID-negative or untested ones (Table 3). Two-thirds of procedures done on COVID-positive patients were upper endoscopies, compared to a third in COVID-negative or untested patients. A higher proportion of endoscopies on COVID-negative patients (29.8\%) were ERCPs compared to COVID-positive patients (10.7\%). COVID-positive patients 
Table 2 Patient characteristics stratified by COVID status

\begin{tabular}{|c|c|c|c|c|}
\hline Variable & COVID-negative & COVID-positive & COVID unknown & $p$-value \\
\hline Total number of patients & $226(41.5 \%)$ & $79(14.5 \%)$ & $240(44.0 \%)$ & \\
\hline Total number of procedures & $255(42.2 \%)$ & $84(13.9 \%)$ & $266(44.0 \%)$ & \\
\hline Median years of age (interquartile range) & $66(55-75)$ & $66(57-76)$ & $60.5(49.5-72.5)$ & 0.006 \\
\hline \multicolumn{5}{|l|}{ Sex } \\
\hline Female & $92(40.7 \%)$ & $21(26.6 \%)$ & $104(43.3 \%)$ & \multirow[t]{2}{*}{0.029} \\
\hline Male & $134(59.3 \%)$ & $58(73.4 \%)$ & $136(56.7 \%)$ & \\
\hline \multicolumn{5}{|l|}{ Admission status } \\
\hline Inpatient & $151(66.8 \%)$ & $72(91.1 \%)$ & $107(44.6 \%)$ & \multirow[t]{2}{*}{$<0.001$} \\
\hline Outpatient & $75(33.2 \%)$ & $7(8.9 \%)$ & $133(55.4 \%)$ & \\
\hline \multicolumn{5}{|l|}{ Month of procedure } \\
\hline March 16-March 31 & $45(17.7 \%)$ & $6(7.1 \%)$ & $209(78.6 \%)$ & \multirow[t]{2}{*}{$<0.001$} \\
\hline April 1-April 30 & $210(82.4 \%)$ & $78(92.9 \%)$ & $57(21.4 \%)$ & \\
\hline \multicolumn{5}{|l|}{ Site of procedure } \\
\hline The Allen Hospital & $4(1.6 \%)$ & $2(2.4 \%)$ & $14(5.3 \%)$ & \multirow[t]{6}{*}{$<0.001$} \\
\hline Columbia University Irving Medical Center & $66(25.9 \%)$ & $16(19.1 \%)$ & $68(25.6 \%)$ & \\
\hline Lower Manhattan Hospital & $12(4.7 \%)$ & $4(4.8 \%)$ & $7(2.6 \%)$ & \\
\hline Mount Sinai Hospital & $84(32.9 \%)$ & $13(15.5 \%)$ & $75(28.2 \%)$ & \\
\hline Montefiore Medical Center & $24(9.4 \%)$ & $14(16.7 \%)$ & $63(23.7 \%)$ & \\
\hline Weill Cornell Medical Center & $65(25.5 \%)$ & $35(41.7 \%)$ & $39(14.7 \%)$ & \\
\hline Outcomes among 330 admitted patients & $N=151$ & $N=72$ & $N=107$ & \\
\hline Admission to Intensive Care Unit & $48(31.8 \%)$ & $47(65.3 \%)$ & $33(30.8 \%)$ & $<0.001$ \\
\hline Intubation & $22(14.6 \%)$ & $39(54.2 \%)$ & $23(21.5 \%)$ & $<0.001$ \\
\hline Death & $10(6.6 \%)$ & $8(11.1 \%)$ & $10(9.4 \%)$ & 0.49 \\
\hline Median length of stay (interquartile range) & $8(4-16)$ & $19(11-29.5)$ & $10(3-26)$ & $<0.001$ \\
\hline
\end{tabular}

Fig. 1 COVID status of patients undergoing endoscopy at 6 academic hospitals in New York City during the peak of the COVID-19 pandemic, March 16 to April 30, 2020

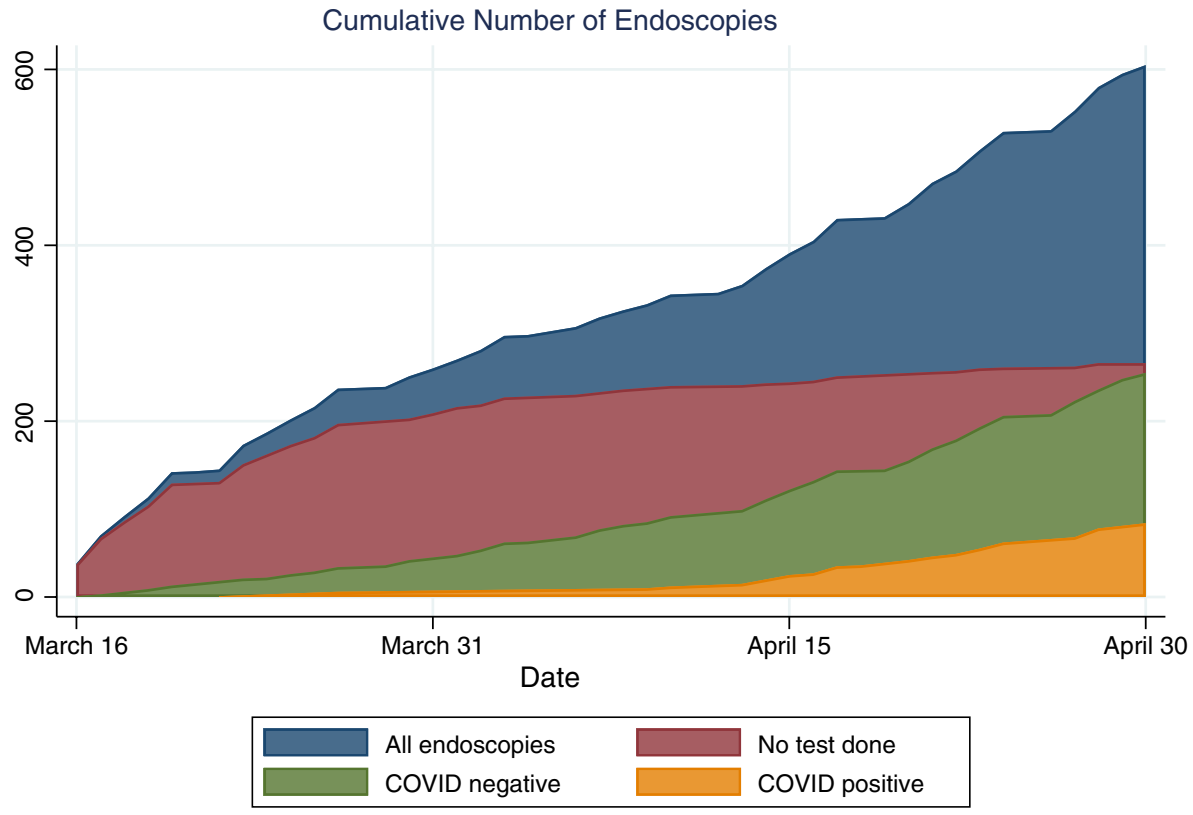

were more likely to undergo endoscopy for gastrointestinal bleeding $(41.7 \%)$ compared to COVID-negative $(29.8 \%)$ or untested patients $(24.1 \%)$. Their procedures were also more likely to be for percutaneous endoscopic gastrostomy tube (PEG) placement or evaluation of dysphagia $(33.3 \%$ of COVID-positive endoscopies, compared to $11.4 \%$ of 
Table 3 Endoscopy characteristics stratified by COVID status

\begin{tabular}{|c|c|c|c|c|}
\hline Variable & COVID-negative & COVID-positive & COVID unknown & $p$ value \\
\hline Procedure type & $N=255$ & $N=84$ & $N=266$ & \\
\hline Upper endoscopy & $96(37.7 \%)$ & $56(66.7 \%)$ & $92(34.6 \%)$ & $<0.001$ \\
\hline Colonoscopy & $22(8.6 \%)$ & $8(9.5 \%)$ & $29(10.9 \%)$ & \\
\hline Small bowel entroscopy & $6(2.4 \%)$ & $1(1.2 \%)$ & $5(1.9 \%)$ & \\
\hline Upper endoscopy + colonoscopy & $16(6.3 \%)$ & $3(3.6 \%)$ & $19(7.1 \%)$ & \\
\hline $\mathrm{ERCP} \pm \mathrm{EUS}$ & $76(29.8 \%)$ & $9(10.7 \%)$ & $63(23.7 \%)$ & \\
\hline EUS alone & $27(10.6 \%)$ & $2(2.4 \%)$ & $44(16.5 \%)$ & \\
\hline Pouchoscopy or flexible sigmoidoscopy & $12(4.7 \%)$ & $5(6.0 \%)$ & $14(5.3 \%)$ & \\
\hline Fellow involvement & $86(33.7 \%)$ & $42(50.0 \%)$ & $114(42.9 \%)$ & 0.014 \\
\hline \multicolumn{5}{|l|}{ Indications $^{\mathrm{a}}$} \\
\hline Gastrointestinal bleeding/anemia & $76(29.8 \%)$ & $35(41.7 \%)$ & $64(24.1 \%)$ & 0.007 \\
\hline Abnormal imaging & $39(15.3 \%)$ & $11(13.1 \%)$ & $30(11.3 \%)$ & 0.4 \\
\hline Biliary obstruction/stone/cholangitis & $66(25.9 \%)$ & $8(9.5 \%)$ & $59(22.2 \%)$ & 0.007 \\
\hline Suspected or known mass & $29(11.4 \%)$ & $1(1.2 \%)$ & $32(12.0 \%)$ & 0.013 \\
\hline Foreign body & $5(2.0 \%)$ & $0(0.0 \%)$ & $3(1.3 \%)$ & 0.495 \\
\hline Inflammatory bowel disease & $4(1.6 \%)$ & $0(0.0 \%)$ & $5(1.9 \%)$ & 0.594 \\
\hline Colorectal cancer screening/surveillance & $4(1.6 \%)$ & $0(0.0 \%)$ & $15(5.6 \%)$ & 0.006 \\
\hline Dysphagia or gastrostomy tube placement & $29(11.4 \%)$ & $28(33.3 \%)$ & $24(9.0 \%)$ & $<0.001$ \\
\hline Gastrointestinal symptoms & $18(7.1 \%)$ & $5(6.0 \%)$ & $23(8.7 \%)$ & 0.655 \\
\hline Stent exchange or removal & $13(5.1 \%)$ & $0(0.0 \%)$ & $11(4.1 \%)$ & 0.114 \\
\hline Pancreatitis & $5(2.0 \%)$ & $0(0.0 \%)$ & $12(4.5 \%)$ & 0.052 \\
\hline Intestinal stenosis/obstruction & $6(2.4 \%)$ & $2(2.4 \%)$ & $5(1.9 \%)$ & 0.921 \\
\hline Other & $4(1.6 \%)$ & $0(0.0 \%)$ & $9(3.4 \%)$ & 0.124 \\
\hline \multicolumn{5}{|l|}{ Anesthesia level } \\
\hline General anesthesia & $123(48.2 \%)$ & $37(44.1 \%)$ & $80(30.1 \%)$ & $<0.001$ \\
\hline Monitored anesthesia care & $114(44.7 \%)$ & $34(40.5 \%)$ & $163(61.3 \%)$ & \\
\hline Moderate sedation & $18(7.1 \%)$ & $13(15.5 \%)$ & $23(8.7 \%)$ & \\
\hline \multicolumn{5}{|l|}{ Endoscopy findings ${ }^{\mathrm{a}}$} \\
\hline Peptic ulcer disease, gastritis, duodenitis, esophagitis & $53(20.8 \%)$ & $26(31.0 \%)$ & $49(18.4 \%)$ & 0.049 \\
\hline Varices, portal hypertensive gastropathy & $14(5.5 \%)$ & $4(4.8 \%)$ & $14(5.3 \%)$ & 0.967 \\
\hline Colon polyps or masses & $15(5.9 \%)$ & $1(1.2 \%)$ & $19(7.1 \%)$ & 0.125 \\
\hline Biliary dilation/stricture, choledocholithiasis, bile leak & $66(25.9 \%)$ & $8(9.5 \%)$ & $53(19.9 \%)$ & 0.005 \\
\hline Blood & $8(3.1 \%)$ & $7(8.3 \%)$ & $7(2.6 \%)$ & 0.044 \\
\hline Extra-colonic nodules, masses, tumors & $35(13.7 \%)$ & $2(2.4 \%)$ & $29(10.9 \%)$ & 0.015 \\
\hline Intestinal stricture/stenosis & $15(5.9 \%)$ & $8(9.5 \%)$ & $22(8.3 \%)$ & 0.428 \\
\hline Colitis & $15(5.9 \%)$ & $7(8.3 \%)$ & $14(5.3 \%)$ & 0.583 \\
\hline Diverticulosis & $11(4.3 \%)$ & $2(2.4 \%)$ & $6(2.3 \%)$ & 0.368 \\
\hline Vascular malformation (AVMs, GAVE, Dieulafoy) & $5(2.0 \%)$ & $1(1.2 \%)$ & $5(1.9 \%)$ & 1 \\
\hline Abscess, fluid collection, cyst & $6(2.4 \%)$ & $2(2.4 \%)$ & $10(3.8 \%)$ & 0.603 \\
\hline Foreign body or food impaction & $4(1.6 \%)$ & $0(0.0 \%)$ & $2(0.8 \%)$ & 0.524 \\
\hline \multicolumn{5}{|l|}{ Maneuvers $^{\mathrm{a}}$} \\
\hline Hemostatic clips & $13(5.1 \%)$ & $11(13.1 \%)$ & $14(5.3 \%)$ & 0.021 \\
\hline Epinephrine injection & $5(2.0 \%)$ & $6(7.1 \%)$ & $4(1.5 \%)$ & 0.012 \\
\hline Bipolar cautery/Argon plasma coagulation/RFA & $8(3.1 \%)$ & $6(7.1 \%)$ & $8(3.0 \%)$ & 0.18 \\
\hline Variceal banding & $6(2.4 \%)$ & $1(1.2 \%)$ & $5(1.9 \%)$ & 0.792 \\
\hline Sphincterotomy, biliary stent, pancreatic stent & $63(24.7 \%)$ & $8(9.5 \%)$ & $44(16.5 \%)$ & 0.003 \\
\hline Forceps biopsy & $39(15.3 \%)$ & $10(11.9 \%)$ & $41(15.4 \%)$ & 0.711 \\
\hline Fine needle aspiration/biopsy & $29(11.4 \%)$ & $1(1.2 \%)$ & $25(9.4 \%)$ & 0.018 \\
\hline Intestinal stent, stricturotomy, or dilation & $19(7.5 \%)$ & $5(6.0 \%)$ & $14(5.3 \%)$ & 0.584 \\
\hline PEG or NG tube placement & $17(6.7 \%)$ & $27(32.1 \%)$ & $11(4.1 \%)$ & $<0.001$ \\
\hline
\end{tabular}


Table 3 (continued)

\begin{tabular}{|c|c|c|c|c|}
\hline Variable & COVID-negative & COVID-positive & COVID unknown & $p$ value \\
\hline Polypectomy or endoscopic mucosal resection & $10(3.9 \%)$ & $0(0.0 \%)$ & $21(7.9 \%)$ & 0.009 \\
\hline Cystogastrostomy or necrosectomy & $5(2.0 \%)$ & $2(2.4 \%)$ & $8(3.0 \%)$ & 0.743 \\
\hline Foreign body or stent removal & $17(6.7 \%)$ & $1(1.2 \%)$ & $24(9.0 \%)$ & 0.047 \\
\hline Video capsule endoscopy placement & $4(1.6 \%)$ & $0(0.0 \%)$ & $3(1.3 \%)$ & 0.679 \\
\hline
\end{tabular}

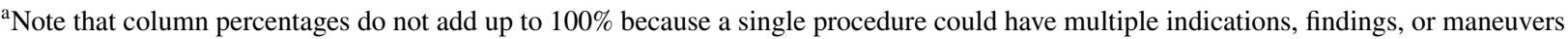

COVID-negative and 9.0\% of COVID untested endoscopies). Figure 2 displays the types of procedures and selected indications done in COVID-positive patients over the study period. COVID-positive procedures were less likely to be for biliary obstruction or suspected or known mass lesions. COVID-positive procedures were significantly more likely to be done in the intensive care unit $29.8 \%$ of COVIDpositive compared to $4.7 \%$ of COVID-negative and $1.9 \%$ of untested endoscopies) or operating room (8.3\% of COVIDpositive compared to $3.9 \%$ of COVID-negative and $3.4 \%$ of untested endoscopies, $p<0.001$ ). A fellow was only involved in $40 \%$ of procedures overall but was more often involved in the COVID-positive cases (50.0\% of COVID-positive compared to $33.7 \%$ of COVID-negative and $42.9 \%$ of untested endoscopies). Confirmed negative COVID procedures were most often done with general anesthesia (48.2\%, compared to $44.7 \%$ monitored anesthesia care and $7.1 \%$ moderate sedation), while untested procedures were most often done with monitored anesthesia care $(61.3 \%$, compared to $30.1 \%$ general anesthesia and $8.7 \%$ moderate sedation). Confirmed positive procedures were also most often done with general anesthesia (44.1\%, compared to $40.5 \%$ monitored anesthesia care) but had a higher proportion than COVID-negative or untested procedures done with moderate sedation (15.5\%). This is likely because many intensive care unit cases were done without anesthesia assistance.

\section{Endoscopy Findings and Interventions}

A higher proportion of COVID-positive procedures found upper gastrointestinal tract ulcers, erosions, or esophagitis, at $31.0 \%$ compared to $20.8 \%$ of COVID-negative and $18.4 \%$ of untested endoscopies. Active bleeding or blood was found in more of the COVID-positive patients as well, at $8.3 \%$ compared to $3.1 \%$ of COVID-negative patients and $2.4 \%$ of untested patients. Masses, biliary strictures, injuries, and stones were found less often in COVID-positive patients. Hemostatic interventions such as clips and epinephrine injections were done more often in COVID-positive patients, though there was no difference in the rate of thermal therapies by COVID status. COVID-positive patients were more likely to require hemostatic interventions even when restricting the analysis to patients undergoing endoscopy for gastrointestinal
Fig. 2 Cumulative numbers of procedures done in COVID-19 patients by type and indication during the peak of the COVID19 pandemic, March 16 to April 30, 2020. Colonoscopy includes flexible sigmoidoscopy, pouchoscopy, and combined upper endoscopy + colonoscopy procedures. ERCP endoscopic retrograde cholangiopancreatography, $P E G$ percutaneous endoscopic gastrostomy tube

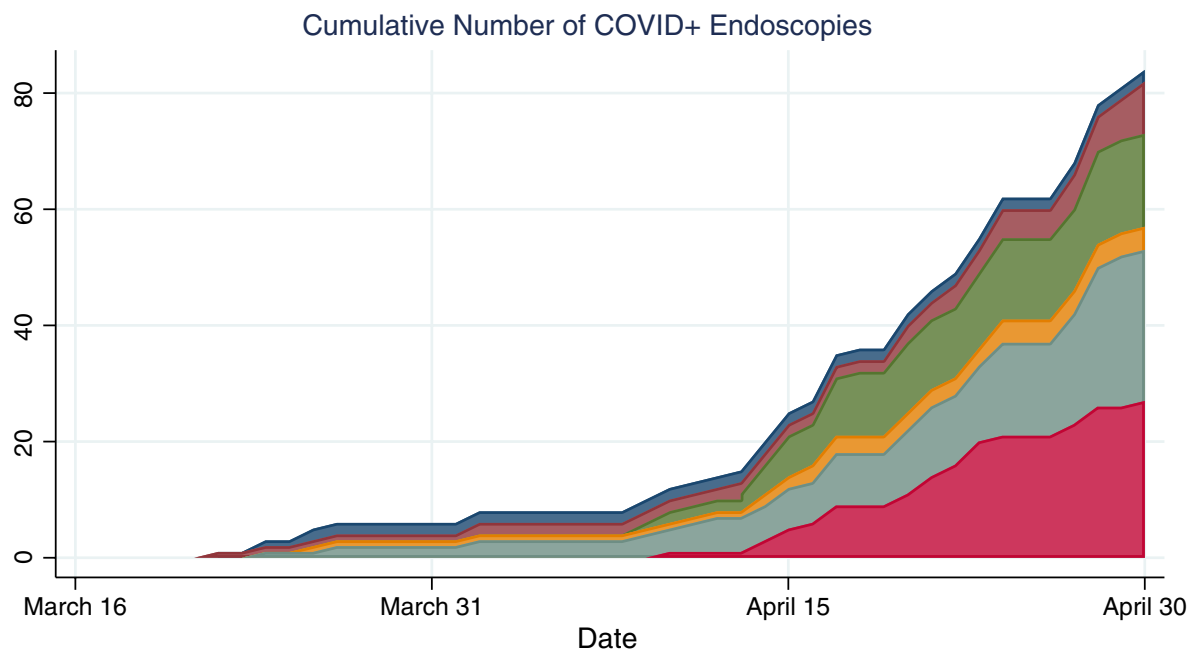

Endoscopic ultrasound

Colonoscopy

Upper endoscopy (GI Bleed)
ERCP

Upper endoscopy (other) Upper endoscopy (PEG) 
bleeding ( $40 \%$ of COVID-positive, $17.1 \%$ of COVIDnegative, and $23.4 \%$ of untested patients, $p=0.032$ ). On multivariable logistic regression analysis, after adjusting for anesthesia level, ICU admission, intubation, age, and sex, a positive COVID test remained significantly associated with the use of hemostatic intervention (adjusted odds ratio 2.90, compared to a negative COVID test, $95 \%$ CI 1.04-8.06, $p=0.041$, Table 4). The receiver operating characteristic (ROC) curve for the model is shown in Supplementary Figure 1, with area under the curve $=0.75$, indicating an acceptable level of predictive accuracy. PEG and endoscopic nasogastric tube placements were also more often done in COVID-positive patients, making up $32.7 \%$ of procedures on COVID-positive patients compared to $6.7 \%$ of COVID-negative and $4.1 \%$ of untested patients $(p<0.001)$. Biliary interventions such as stents, sphincterotomy, and stone extractions, as well as fine needle aspiration or biopsy, were done less often in the COVID-positive patients, because ERCPs were done less often. Among just those patients undergoing ERCP, COVID-patients had a similar rate of biliary or pancreatic stent placement (88.9\% in COVID-positive patients, $79.0 \%$ in COVID-negative patients, $p=0.21$ ).

\section{Patients Who Tested Positive for COVID After Endoscopy}

There were 27 patients who tested positive for COVID only after their endoscopy (Supplementary Table 3), at a median of 13.5 days post-endoscopy (interquartile range 7-24). Three of the 27 (11\%) tested negative prior to endoscopy and subsequently tested positive a median of days postendoscopy, while 24 (89\%) had no testing before their first endoscopy and later tested positive a median of 14 days postendoscopy. There were 30 endoscopies done on these 27 patients before they tested positive, and 22 of the 27 were inpatient. Compared to procedures done on patients who never tested positive or who were always positive, procedures done on patients who subsequently became positive were more likely to be a colonoscopy $(8.7 \%$ and $9.5 \%$ compared to $20 \%$, respectively) or an upper endoscopy with colonoscopy (6.1\% and 3.6\% compared to $10 \%$, respectively). Most of the patients who were found to be positive after endoscopy were initially endoscoped in March, when testing capability was more limited (73.3\%, compared to $7.1 \%$ of the known COVID-positive patients, $p<0.001$ ). Compared to patients who were known to be positive prior to endoscopy, patients diagnosed after their procedure were more likely to be outpatient ( $26.7 \%$ compared to $8.3 \%, p=0.011$ ), and
Table 4 Multivariable logistic regression model for hemostatic intervention among patients undergoing endoscopy for gastrointestinal bleeding during the COVID-19 pandemic

\begin{tabular}{|c|c|c|c|c|}
\hline Variable & $\begin{array}{l}n \text { who received } \\
\text { hemostasis } / n \text { endo- } \\
\text { scoped }\end{array}$ & Adjusted odds ratio & $\begin{array}{l}95 \% \text { confi- } \\
\text { dence interval }\end{array}$ & $p$ value \\
\hline \multicolumn{5}{|l|}{ COVID status } \\
\hline Negative & $13 / 76(17.1 \%)$ & 1 (reference) & & \\
\hline Positive & $14 / 35(40.0 \%)$ & 2.90 & $1.04-8.06$ & 0.041 \\
\hline Unknown & $15 / 64(23.4 \%)$ & 1.86 & $0.75-4.65$ & 0.18 \\
\hline \multicolumn{5}{|l|}{ Intensive care unit admission } \\
\hline No & $23 / 103(22.3 \%)$ & 1 (reference) & & \\
\hline Yes & $19 / 72(26.4 \%)$ & 0.46 & $0.13-1.59$ & 0.22 \\
\hline \multicolumn{5}{|l|}{ Intubation } \\
\hline No & $27 / 129(20.9 \%)$ & 1 (reference) & & \\
\hline Yes & $15 / 46(32.6 \%)$ & 3.37 & $0.86-13.17$ & 0.081 \\
\hline \multicolumn{5}{|l|}{ Sex } \\
\hline Female & $11 / 55(20.0 \%)$ & 1 (reference) & & \\
\hline Male & $31 / 120(25.8 \%)$ & 1.11 & $0.46-2.67$ & 0.81 \\
\hline \multicolumn{5}{|l|}{ Age } \\
\hline$<50$ & $5 / 31(16.1 \%)$ & 1 (reference) & & \\
\hline $50-59$ & $5 / 38(13.2 \%)$ & 0.61 & $0.14-2.64$ & 0.51 \\
\hline $60-69$ & $12 / 43(27.9 \%)$ & 2.34 & $0.63-8.74$ & 0.20 \\
\hline$\geq 70$ & $20 / 63(31.8 \%)$ & 4.39 & $1.26-15.30$ & 0.020 \\
\hline \multicolumn{5}{|l|}{ Anesthesia type } \\
\hline Moderate sedation & 4/21 (19.1\%) & 1 (reference) & & \\
\hline Monitored anesthesia care & $22 / 115(19.1 \%)$ & 1.43 & $0.39-5.21$ & 0.59 \\
\hline General anesthesia & $16 / 39(41.0 \%)$ & 6.24 & $1.47-26.43$ & 0.013 \\
\hline
\end{tabular}


less likely to undergo upper endoscopy ( $30.0 \%$ compared to $66.7 \%, p=0.037)$. They were more likely to undergo endoscopy for colorectal cancer screening or surveillance (13.3\% compared to $0.0 \%$ ). Among the 383 hospitalized patients, those who tested positive only after endoscopy had the highest mortality rate at $31.8 \%$ (7/22 patients), compared to $8.5 \%$ in patients who only tested negative, and $11.7 \%$ in patients who were positive prior to endoscopy $(p=0.001)$.

\section{Discussion}

This multicenter study describes the characteristics and outcomes of all adult patients undergoing endoscopy at six academic hospitals in New York City during the peak of the COVID-19 pandemic in early 2020. As described in a New York Society of Gastrointestinal Endoscopy survey, the number of routine endoscopy cases significantly decreased in our region during the pandemic [2]. Many gastroenterologists stopped performing endoscopies completely during the peak of the pandemic. The procedures deemed urgent enough to be performed during this period were generally on patients more seriously ill than what is typical.

All six institutions in this study adopted similar protocols in regard to COVID testing and personal protective equipment utilization. At all institutions, routine endoscopy was discontinued starting March 16, 2020. COVID testing was initially not widely available, but beginning in late March was performed first on patients suspected of having COVID and eventually universally on all patients undergoing endoscopy. Negative pressure rooms were utilized for all confirmed COVID-positive patients. Personal protective equipment consisted of at minimum an $\mathrm{N} 95$ respirator, gown, face shield, and hair cover, and at some institutions shoe covers were also required.

Our findings illustrate the dramatic increase in testing that occurred toward the end of March. Initially, almost all endoscopies were done without testing, but over time as tests became more available nearly all endoscopies were done with pre-procedure testing. Figure 1 and Supplementary Figure 2 show the trend toward increased testing over the course of the pandemic. Though patients were screened for symptoms such as cough or fever, it is now well known that many patients are asymptomatic carriers of SARS-CoV-2 [9]. In fact, 22 patients who had a procedure without testing were subsequently found to test positive. It is clear that screening for symptoms is not a rigorous enough method for limiting unnecessary exposures during endoscopy. Gastroenterologists in areas that are still experiencing the peak of the COVID-19 pandemic should be aware that they may be performing procedures on patients with undiagnosed COVID19 if pre-procedure testing is inadequate. These findings are particularly important in light of evidence that shows that viral RNA is consistently detectable in secretions commonly encountered during gastrointestinal procedures including saliva and stool, and that detection of the viral shedding in stool may persist following clearance of virus from aerodigestive tract samples $[10,11]$. A recent study also provides evidence that EGDs are aerosol generating procedures [12]. Patients who were later diagnosed with COVID were more likely to have outpatient procedures compared to patients who were already known to have COVID, so it is important not to limit testing to inpatients. Economic analysis has suggested that universal pre-endoscopy COVID PCR testing is a cost-effective strategy for reopening endoscopy units, and our findings add further weight to this policy, which has already been implemented in many centers [13]. In addition to screening for COVID, we suggest providers use of N95 respirators and face shields in addition to standard personal protective equipment, restrict the number of unnecessary personnel, and carefully weigh the risks and benefits of endoscopic procedures, in order to limit transmission of coronavirus as much as possible.

We further noted that clinical outcomes, including mortality, of patients who only tested positive after endoscopy were significantly worse, even compared to patients who were known to be COVID-positive at the time of endoscopy. The sample size of patients diagnosed with COVID only after endoscopy was small, and it is unclear why they should have a higher mortality than patients who were known to be positive. It is possible that undiagnosed COVID could have been associated with gastrointestinal symptoms prompting endoscopy, though the number of patients endoscoped for gastrointestinal symptoms was low. It is also possible that procedural sedation early in the disease in patients with undiagnosed COVID could lead to downstream harm. There may be unmeasured confounders as well, such as race and ethnicity, which are associated with both lack of early testing and poor outcomes. Delays in diagnosis of COVID are associated with worse outcomes and likely also contributed to the higher mortality rate in patients diagnosed post-endoscopy.

Surprisingly, there were 19 colonoscopies done for screening or surveillance during the peak of the pandemic, 4 on COVID-negative patients and 15 on patients without COVID testing. In three of these cases, another indication such as GI bleeding was also listed. In the other 16 cases, it is possible that additional undocumented indications led to the decision to pursue a colonoscopy during the pandemic, but it is also possible that a small number of less urgent colonoscopies were still performed for screening, though a small fraction of the number that would ordinarily be performed during this time period. We believe that any unreliability in documented Provation indications would be primarily limited to colonoscopies, in which screening is often combined with a secondary indication. 
Another surprising finding was that trainees, specifically fellows, were more likely to be involved in COVID-positive cases $(50.0 \%$ compared to $33.7 \%$ of COVID-negative and $42.9 \%$ of untested patients). Fellows were more often involved in EGDs, which were the most common procedures done in COVID patients. This may be because a second operator is helpful for PEG placement and GI bleeding cases, whereas for cases such as colonoscopies a single endoscopist is sufficient. For example, GI bleeding cases are often technically complex and at times done overnight or in the ICU, where an endoscopy nurse or technician may not be available. In those cases, a fellow may be helpful for providing assistance when a second attending is not available. Over time, as COVID testing became more common, fellows started to become involved again, perhaps because redeployed fellows were again available for procedures, and as a result of greater availability of PPE. Supplementary Figure 3 shows the numbers of fellow and non-fellow cases by week.

This study describes the diagnostic yield, rate of interventions, and clinical outcomes of patients undergoing endoscopy with COVID-19 compared to those testing negative and those without testing. Endoscopies done in patients with COVID-19 were more often done for gastrointestinal bleeding. This could be a consequence of the increasing use of anticoagulation in patients with COVID-19, or the fact that while most gastroenterologists have a higher threshold to scope COVID-positive patients, gastrointestinal hemorrhage is one of the indications that cannot typically be deferred $[14,15]$. It is also likely a result of the fact that COVID patients were more likely to be admitted to the ICU, and ICU patients in general are at higher risk of peptic ulcer disease. PEG or nasogastric tube placement was also very common in COVID-19 patients, reflecting the unfortunate reality that many patients with COVID-19 face a long recovery after extubation. Approximately a third of endoscopies done in COVID-positive patients were for PEG or NG tube placement, likely because elective procedures were avoided as much as possible in COVID patients, who were also more likely to require prolonged intubation leading to dysphagia.

The diagnostic yield of procedures done during this period was high. For example, of the 175 endoscopies done for anemia or bleeding, $73.1 \%$ found blood or a possible source such as ulcers, erosions, esophagitis, arteriovenous malformations, or diverticulosis. The yield was particularly high among the 35 endoscopies done for bleeding in COVID-positive patients, at $85.7 \%$, though due to the low numbers this was not significantly different from that of COVID-negative $(69.7 \%)$ or untested patients $(70.3 \%)$. Endoscopies done for anemia or bleeding in COVID-positive patients had about twice the rate of hemostatic clip, epinephrine, or electrocoagulation use $(14 / 35,40.0 \%)$ compared to COVID-negative $(13 / 76,17.1 \%)$ or untested patients $(15 / 64$,
$23.4 \%, p=0.032$ ), likely reflecting a higher clinical threshold for pursuing endoscopy in COVID-positive patients.

This study is the largest in-depth analysis of patients undergoing endoscopy during the COVID-19 pandemic in the USA and reflects the beginning and peak of the pandemic. Strengths of this study are the multicenter design and setting in the early epicenter of the COVID-19 pandemic after the arrival of the virus in the USA in 2020. A limitation is the relatively short follow-up time available to measure clinical outcomes such as intensive care unit admission, intubation, death, and length of stay. This was necessary to avoid delays in analysis, and as a result we may be underestimating these outcomes in this cohort. Further, testing was limited in the early phase of the pandemic partially due to lack of adequate supplies. The sensitivity of the SARS-CoV-2 PCR is also suboptimal and likely resulted in some false negative results [16]. Analysis was generally limited to variables extracted from the Provation endoscopy software, and some clinically relevant variables such as medications (including anticoagulation) race, and cause of death were not obtained. Finally, due to the design of this study only patients with indications urgent enough to undergo endoscopy during the pandemic were evaluated, and we did not investigate patients who were treated conservatively.

In summary, while endoscopies continued to be performed during the COVID-19 pandemic in our region, the risk-benefit ratio shifted, and cases were generally limited to urgent or emergent indications. The patients who meet these stricter criteria are sicker, especially the ones who are known to be COVID-positive, and the in-hospital mortality rate among COVID patients undergoing endoscopy is high. Patients undergoing endoscopy for gastrointestinal bleeding with COVID are more likely to require hemostatic interventions, likely because we are limiting endoscopy in that population to patients with a high suspicion for active hemorrhage. It is important to recognize that many patients who underwent endoscopy before testing was widely available were subsequently found to test positive for COVID, and thus universal testing prior to endoscopy should be pursued to limit unnecessary exposures.

Author's contribution Study concept and design were done by JWB, DEF, BL, NAK, RGC, SH, TAG, SM. Data collection was done by JWB, NAK, RED, YD, SN, CJD, DG, RZS, DCL, AGG, SH, AR, VA, KS, RGC, SM. Analysis and Interpretation were done by JWB, DEF, SM, BL, NAK, SH, KS, SN, DCL, SH, TAG. Manuscript preparation was done by JWB, DEF, SM, DCL, NAK, KS, BL. Critical revision of the manuscript was done by JWB, NAK, RED, YD, SN, CJD, DG, RZS, DCL, AGG, SH, BL, RGC, AR, VA, TAG, DEF, SM, KS. Project supervision was done by NAK, SH, TAG, DEF, SM. Final Approval was done by JWB, NAK, RED, YD, SN, CJD, DG, RZS, DCL, AGG, SH, AR, VA, KS, RGC, BL, TAG, DEF, SM.

Funding None. 


\section{Compliance with Ethical Standards}

Conflict of interest C.J.D.: Boston Scientific: consultant, teaching, Medtronic: consultant, teaching, AbbView: speaking. N.A.K.: Consultant: Apollo Endosurgery, Boston Scientific, Gyrus Acmi Inc, Olympus. All other authors have no conflicts of interest to disclose.

Ethical approval All procedures performed in studies involving human participants were in accordance with the ethical standards of the institutional and/or national research committee and with the 1964 Helsinki declaration and its later amendments or comparable ethical standards.

Informed consent For this type of study formal consent is not required.

\section{References}

1. COVID-19 United States Cases by County. Johns Hopkins University, https://coronavirus.jhu.edu/us-map. Accessed 25 Apr 2020.

2. Mahadev S, Aroniadis OS, Barraza L, et al. Impact of the COVID19 pandemic on endoscopy practice: results of a cross-sectional survey from the New York metropolitan area. Gastrointest Endosc. 2020. https://doi.org/10.1016/j.gie.2020.04.047.

3. Forbes NSZ, Spitzer RL, Keswani RN, Wani SB, Elmunzer BJ. Changes in gastroenterology and endoscopy practices in response to the COVID-19 pandemic: results from a North American survey. Gastroenterology. 2020. https://doi.org/10.1053/j.gastr o.2020.04.071.

4. Kim J, Doyle JB, Blackett JW, et al. Effect of the COVID-19 pandemic on outcomes for patients admitted with gastrointestinal bleeding in New York City. Gastroenterology. 2020. https://doi. org/10.1053/j.gastro.2020.05.031.

5. Soetikno R, Teoh AY, Kaltenbach T, et al. Considerations in performing endoscopy during the COVID-19 pandemic. Gastrointest Endosc. 2020. https://doi.org/10.1016/j.gie.2020.03.3758.

6. Danese S, Ran ZH, Repici A, et al. Gastroenterology department operational reorganisation at the time of covid-19 outbreak: an Italian and Chinese experience. Gut. 2020. https://doi. org/10.1136/gutjnl-2020-321143.
7. Repici A, Maselli R, Colombo M, et al. Coronavirus (COVID-19) outbreak: what the department of endoscopy should know. Gastrointest Endosc. 2020. https://doi.org/10.1016/j.gie.2020.03.019.

8. Repici A, Pace F, Gabbiadini R, et al. Endoscopy units and the COVID-19 outbreak: a multi-center experience from Italy. Gastroenterology. 2020. https://doi.org/10.1053/j.gastro.2020.04.003.

9. Bai Y, Yao L, Wei T, et al. Presumed asymptomatic carrier transmission of COVID-19. JAMA. 2020. https://doi.org/10.1001/ jama.2020.2565.

10. Xiao F, Tang M, Zheng X, Liu Y, Li X, Shan H. Evidence for gastrointestinal infection of SARS-CoV-2. Gastroenterology. 2020;158:1831-3 e3. https://doi.org/10.1053/j.gastro.2020.02.055.

11. To KK, Tsang OT, Chik-Yan Yip C, et al. Consistent detection of 2019 novel coronavirus in saliva. Clin Infect Dis. 2020. https:// doi.org/10.1093/cid/ciaa149.

12. Chan SM, Ma TW, Ka-Chun Chong M, Chan DL, Ng EKW, Chiu PWY. A proof of concept study: esophagogastroduodenoscopy is an aerosol-generating procedure and continuous oral suction during the procedure reduces the amount of aerosol generated. Gastroenterology. 2020. https://doi.org/10.1053/j.gastro.2020.07.002.

13. Corral JE, Hoogenboom SA, Kroner PT, et al. COVID-19 polymerase chain reaction testing before endoscopy: an economic analysis. Gastrointest Endosc. 2020. https://doi.org/10.1016/j. gie.2020.04.049.

14. Tang N, Bai H, Chen X, Gong J, Li D, Sun Z. Anticoagulant treatment is associated with decreased mortality in severe coronavirus disease 2019 patients with coagulopathy. J Thromb Haemost. 2020;18:1094-1099. https://doi.org/10.1111/jth.14817.

15. Kollias A, Kyriakoulis KG, Dimakakos E, Poulakou G, Stergiou GS, Syrigos K. Thromboembolic risk and anticoagulant therapy in COVID-19 patients: emerging evidence and call for action. $\mathrm{Br}$ J Haematol. 2020. https://doi.org/10.1111/bjh.16727.

16. Drame M, Teguo MT, Proye E, et al. Should RT-PCR be considered a gold standard in the diagnosis of Covid-19? J Med Virol. 2020. https://doi.org/10.1002/jmv.25996.

Publisher's Note Springer Nature remains neutral with regard to jurisdictional claims in published maps and institutional affiliations. 